\title{
Increased robustness and speed in low-dose phase-contrast tomography with laboratory sources
}

\author{
Anna Zamir ${ }^{\mathrm{a}}$, Charlotte K Hagen ${ }^{\mathrm{a}}$, Paul C Diemoz ${ }^{\mathrm{a}}$, Marco Endrizzi ${ }^{\mathrm{a}}$, Fabio A Vittoria ${ }^{\mathrm{a}}$, Luca \\ Urbani $^{\mathrm{b}}$, Paolo De Coppi ${ }^{\mathrm{b}}$, and Alessandro Olivo ${ }^{\mathrm{a}}$ \\ ${ }^{a}$ Department of Medical Physics and Biomedical Engineering, University College London, \\ London WC1E 6BT, UK \\ ${ }^{\mathrm{b}}$ Institute of Child Health and Great Ormond Street Hospital, University College London, \\ London WC1N 1EH, UK
}

\begin{abstract}
In this article we discuss three different developments in Edge Illumination (EI) X-ray phase contrast imaging (XPCi), all ultimately aimed at optimising EI computed tomography (CT) for use in different environments, and for different applications. For the purpose of reducing scan times, two approaches are presented; the "reverse projection" acquisition scheme which allows a continuous rotation of the sample, and the "single image" retrieval algorithm, which requires only one frame for retrieval of the projected phase map. These are expected to lead to a substantial reduction of EI CT scan times, a prospect which is likely to promote the translation of EI into several applications, including clinical. The last development presented is the "modified local" phase retrieval. This retrieval algorithm is specifically designed to accurately retrieve sample properties (absorption, refraction, scattering) in cases where high-resolution scans are required in non-ideal environments. Experimental results, using both synchrotron radiation and laboratory sources, are shown for the various approaches.
\end{abstract}

Keywords: Phase-contrast imaging, edge illumination, computed tomography, fast imaging, phase retrieval, X-ray imaging

\section{INTRODUCTION}

In X-ray Phase Contrast imaging (XPCi), images offer access to information about the refraction and ultra-small angle scattering (USAXS) properties of a sample, in addition to the absorption data provided by conventional Xray radiography. ${ }^{1,2}$ While most XPCi methods require a high brilliance X-ray source, both the Edge Illumination (EI) and Grating Interferometry (GI) methods can be additionally used with low brilliance sources (e.g. X-ray tubes), making their translation to a clinical environment more likely. ${ }^{3-11}$ In contrast to GI which relies on the interference of X-rays and so requires phase and absorption gratings' periods of few $\mu m$, the EI method, which is the focus of this article, is totally non-interferometric and its sensitivity is independent of the gratings' period, enabling larger and more robust setups. ${ }^{8}$

As demonstrated in Fig. 1, the sensitivity to refraction in EI is realized by the use of two gratings (hereafter called "masks"): one placed before the sample ("sample mask") and the other immediately before the detector ("detector mask"). While the sample mask shapes the incoming X-ray beam into a single laminar beam (in the synchrotron implementation, see Fig. 1(a)) or multiple, separate beamlets (in the laboratory implementation, see Fig. 1(b)), the detector mask is positioned such that it covers parts of the detector pixels. The sensitivity to refraction arises from aligning the masks in such a way that only part of the beam transmitted through the sample mask reaches the active area of the detector pixel (hence "Edge Illumination"). This way, refraction caused by a sample will be translated into an increase/decrease in detected intensity as it shifts the beam towards/away from the exposed pixel.

EI was first developed as a synchrotron modality, with the sample mask comprising of a single slit (therefore requiring scanning of the sample). ${ }^{3}$ While this is feasible for high-flux sources such as those found in

Send correspondence to A.Z.: E-mail: anna.zamir.10@ucl.ac.uk

Developments in X-Ray Tomography X, edited by Stuart R. Stock, Bert Müller, Ge Wang, Proc. of SPIE Vol. 9967, 996716 · (c) 2016 SPIE · CCC code: 0277-786X/16/\$18 · doi: 10.1117/12.2236626 
synchrotrons, the laboratory implementation of EI involves the use of masks with multiple slits, such that the edge-illumination condition is met for each detector row/column. ${ }^{4}$ While slits allow for phase sensitivity in one direction only, more complex aperture designs can be used to obtain two-directional sensitivity. ${ }^{12,13}$ In EI, a single projection of a sample contains mixed information about its refraction, absorption and scattering distribution. However, dedicated algorithms ("phase retrieval" algorithms) have been developed to obtain separate refraction and absorption projections from two mixed frames, plus a USAXS projection if a third frame is acquired. ${ }^{6,14,15}$ Systems sensitive to phase in two directions typically require more than two or three frames for the retrieval. ${ }^{13}$ The spatial resolution in a projection can be varied; while the period of the sample mask dictates the spatial sampling rate in a single projection, the spatial resolution can be artificially increased by a process called "dithering". When dithering is employed, the sample is translated by sub-period steps, and projections are acquired at each step. These projections are then recombined to create a higher resolution projection, albeit at the cost of increased dose and acquisition time.

The EI method has been also extended to computed tomography (CT) and has been shown to provide quantitative information about a sample's complex refractive index, with both synchrotrons and conventional X-ray sources. ${ }^{16,17}$ For the latter, this is true within the limits imposed by the use of a polychromatic source, as explained in detail in a publication by Munro and Olivo ${ }^{18}$ and experimentally validated and discussed by Hagen et al. (see supplementary material in the publication). ${ }^{19}$ EI offers flexible imaging, as depending on the imaged sample and its application, acquisitions can be altered such that either the spatial resolution is maximized, or the dose is reduced. ${ }^{6,17,19,20}$ Together with its suitability for different imaging environments, EI therefore has the potential to be a valuable tool for research in a wide range of applications, and indeed recent experiments ranged from the biomedical field, to material science and homeland security. ${ }^{21-24}$

Currently, two different requirements are being considered as prospective developments in EI CT. The first is fast imaging, possibly at the expense of an additional contrast channel (e.g. scattering distribution) or spatial resolution. The second is the requirement for high resolution CT scans, which are inevitably associated with long exposures, often leaving the imaging setup exposed to environmental effects such as vibrations or temperature changes, which can in turn lead to artefacts in the reconstructed image. In the following, we discuss two recently developed approaches ("reverse projection", "single image retrieval") aimed to fulfill the requirement for fast imaging, and the "modified local retrieval" method, which was designed to reduce the artefacts associated with long, high-resolution CT scans in non-ideal environments. All these are promising developments, likely to increase the potential of translating the EI technique into clinics while maintaining its applicability to diverse fields.

\section{MATERIALS AND METHODS}

\subsection{Reverse projection}

As mentioned above, separate absorption and refraction projections can be retrieved in EI, by use of phase retrieval algorithms, amongst which the most commonly used are the ones developed by Munro et al. and Diemoz et al. 8,14 These specific algorithms (here called "two-image retrieval"), which neglect sample scattering, require a preliminary measurement of the illumination curve (IC). The IC (see Fig.2(a)) is obtained by recording the detected intensity as a function of sample mask position, while the sample mask is moved over one period. The retrieval algorithm relies on the fact that while the absorption signal of two projections acquired at opposite points on the IC's slopes (see blue and red circles in Fig.2(a)) will remain identical, the refraction signal will reverse. Therefore, normally in EI CT, at each angular position of the sample (over a $180^{\circ}$ range), two projections are acquired corresponding to opposite illumination configurations (as shown for a single pixel in Fig.2(b,c)). This is realized by repositioning the sample mask between the two projections, therefore adding "dead time" to the acquisition. The reverse projection concept, first described by Wang et al. for diffraction enhanced imaging, ${ }^{25}$ removes the need for mask movement between projections, therefore reducing scan time. Here, instead of acquiring two frames per angular position over a $180^{\circ}$ range, the sample is rotated over $360^{\circ}$, while the sample mask is kept in the same position throughout the scan. This acquisition scheme is equivalent to the one described previously, since the absorption signal remains the same for two projections separated by $180^{\circ}$,

Proc. of SPIE Vol. $9967996716-2$ 
a)

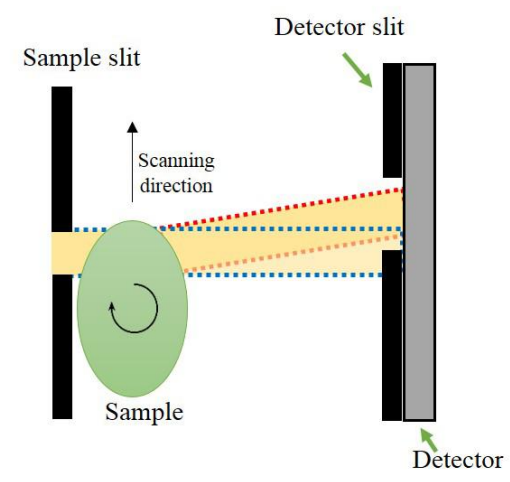

b)

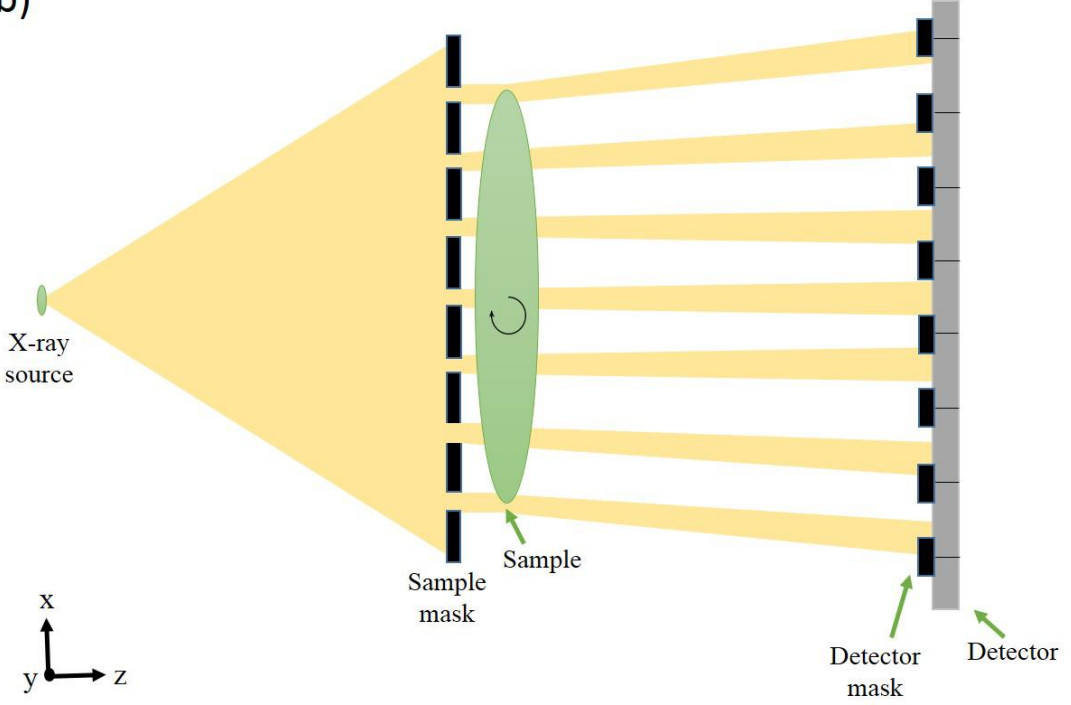

Figure 1. Schematic of the EI principle. (a) In the synchrotron implementation, both the sample and detector masks comprise of a single slit, hence requiring scanning of the sample in the direction perpendicular to the slit. Sensitivity to refraction arises from the difference in detected intensity in the presence of a sample (red dotted line), and without a sample (blue dotted line). (b) In the laboratory implementation, masks with multiple slits are used for area imaging.

while the refraction signal reverses (see Fig.2(b,d)). Therefore, projections separated by $180^{\circ}$ can be processed together, using the same two-image phase retrieval algorithm mentioned previously, however without the need for moving the sample mask. ${ }^{26}$ The theoretical background has been developed and adapted to the EI setup, showing that the refraction angle $\alpha(x, \theta)$ can be retrieved according to:

$$
\alpha(x, \theta)=F^{-1}\left(\frac{P(M x, \theta)}{P(-M x, \theta+\pi)}\right)
$$

where $x$ is the sample coordinate in the direction perpendicular to the masks apertures, $\theta$ is the sample rotation angle, and $P$ is a projection acquired for a given sample position and rotation. $M$ is the magnification between the sample and detector planes, and $F^{-1}$ is the inverse of the function $F$ described by:

$$
F(\alpha):=\frac{C\left(x_{m 1}+z \alpha / M\right)}{C\left(x_{m 1}-z \alpha / M\right)}
$$

which can be calculated from the illumination curve $(C)$, knowing the position of the sample mask $\left(x_{m 1}\right)$, the sample-to-detector distance $(z)$ and the magnification $(M)$. The sample attenuation $A(x, \theta)$ is then calculated from:

$$
A(x, \theta)=\ln \left(\frac{P(M x, \theta)}{C\left(x_{m 1}+z \alpha(x, \theta) / M\right)}\right) .
$$

Quantitative and qualitative validation of the reverse projection method was carried out in our laboratory by scanning both a biological sample (fresh chicken wing, using dithering) and a custom-built phantom (containing oil and water, employing a continuous sample rotation, without dithering). ${ }^{26}$ Here, we report results from a laboratory scan with a total exposure time of approximately 14 minutes. The CT scan was performed using a Rigaku MicroMax 007 HF rotation anode (Molybdenum) X-ray tube (Rigaku Corporation, Japan), with a focal spot measured as approximately $70 \mu \mathrm{m}$ horizontally, and operated at $35 \mathrm{kVp}$ and $25 \mathrm{~mA}$. The detector 
a)

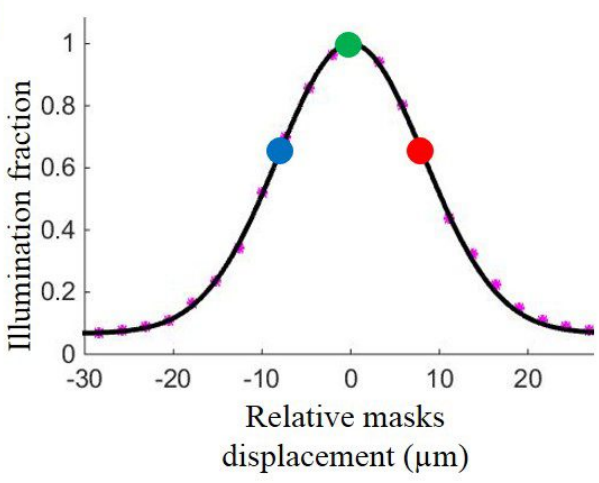

c)

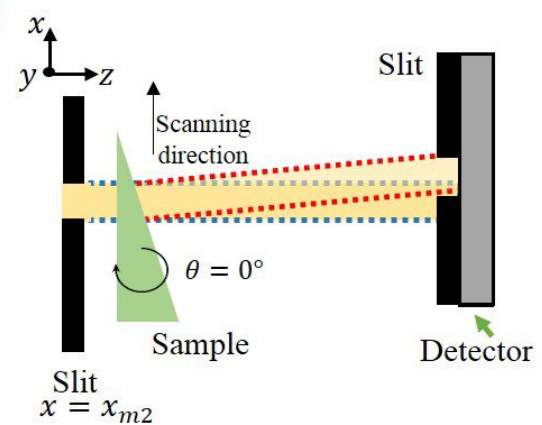

b)

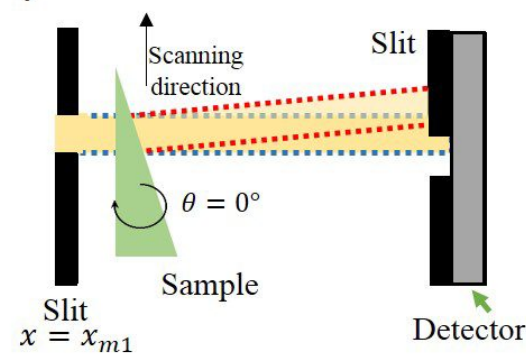

d)

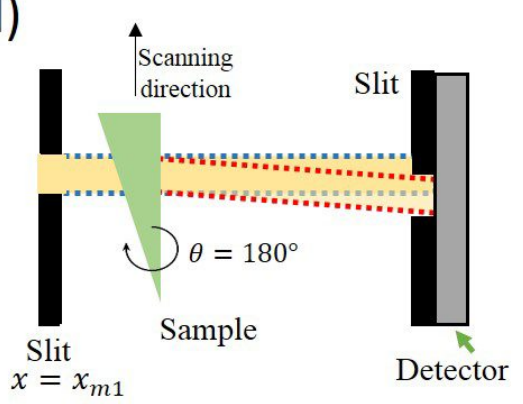

Figure 2. (a) A typical illumination curve (IC) showing the variation in detected intensity as a function of relative masks displacement. The magenta stars mark the experimentally measured points, while the solid black line is the fitted illumination curve. The blue and red dots mark the positions of opposite illumination configurations chosen for imaging using the conventional two-image retrieval algorithm, corresponding to the setups shown in panels (b,c). If a third frame is required (for retrieval of the scattering distribution), another image can be taken where the masks' apertures overlap, as marked by the green circle in (a). For the reverse projection method, the sample mask will remain in the same position (e.g. blue dot), and projections separated by $180^{\circ}$ will be processed together, as shown in panels (b,d).

was a Hamamatsu C9732DK flat panel, a passive-pixel CMOS sensor (Hamamatsu, Japan), with a pixel size of $50 \times 50 \mu \mathrm{m}^{2}$. The detector was located at $2 \mathrm{~m}$ from the source, and the effective pixel size in the horizontal direction was $100 \mu \mathrm{m}$, due to the period of the detector mask, designed to decrease pixel cross-talk. The sample mask (aperture width $=23 \mu \mathrm{m}$, period $=79 \mu \mathrm{m}$ ) and detector mask (aperture width $=29 \mu \mathrm{m}$, period $=98 \mu \mathrm{m}$ ) were positioned at $1.6 \mathrm{~m}$ and $1.96 \mathrm{~m}$ downstream the source, respectively. The sample (a dung beetle, placed $5 \mathrm{~cm}$ downstream the sample mask) was scanned over $360^{\circ}$, with an angular step of $0.5^{\circ}$. At each rotation angle, two images were acquired at opposite illumination configurations, to enable a direct comparison of the reverse projection method with the one previously described. The exposure time per projection was $1.2 \mathrm{~s}$. For phase retrieval, the dataset was then processed twice, once using the conventional method (two frames per rotation angle, using projections acquired over a $180^{\circ}$ rotation), and once with the reverse projection method (using one frame per rotation angle, for rotation over $360^{\circ}$ ). Separate absorption and differential phase sinograms were created, and $\mathrm{CT}$ reconstruction was performed using the filtered back-projection method.

\subsection{Single image retrieval}

While the reverse projection method described above has the potential to reduce scan times by allowing a continuous rotation of the sample, the total number of required frames remains the same as in the conventional EI retrieval (i.e. two frames are processed together to obtain separate absorption and refraction images). The single image retrieval could, in principle, have the potential to halve scan times since, as the name suggests, it 
requires a single input frame per projection. ${ }^{27}$ Here, the detected signal is expressed as a function combining expressions for the EI signal in one direction (perpendicular to the slit), and free-space propagation signal ${ }^{20}$ in the other (parallel to the slits). For a homogeneous sample of known material and small refraction angles, the developed equation can be used to accurately retrieve the sample's projected thickness, using a single projection. However, the assumption of a homogeneous material can be relaxed, and instead we can assume that the sample has a constant ratio, $\gamma$, between the real and imaginary parts of its complex refractive index, as is often the case for biological samples containing several different soft tissues (an assumption previously made in various algorithms $\left.{ }^{28-30}\right)$. Using this approach, an expression was developed to obtain the projected electron density, which is directly proportional to the phase. Therefore, the sample's phase map can be directly retrieved from a single EI image.

The theoretical background has been derived, and the algorithm's quantitative accuracy was validated using synchrotron radiation and a custom-built wire phantom. ${ }^{27}$ Here, we show example results from a scan of bamboo wood (approximately $500 \mu \mathrm{m}$ thick) performed using synchrotron radiation at the I13 beamline of the Diamond synchrotron radiation facility (Didcot, UK). The X-ray energy was $9.7 \mathrm{keV}$, and the detector used was PCO Edge camera, consisting of a scintillator, magnifying visible light optics and a sCMOS sensor, with an effective pixel size at the sample plane of $0.8 \mu \mathrm{m}$. The sample slit $(3 \mu \mathrm{m}$ horizontal aperture) was positioned 5 $\mathrm{cm}$ upstream the sample, and the sample-to-detector distance was $30 \mathrm{~cm}$. Here, a detector slit was not used, and instead the "virtual edge" EI configuration was employed. ${ }^{31}$ The sample was scanned with a $1.6 \mu \mathrm{m}$ step. The EI image was then processed according to the proposed algorithm, using an estimated value of $\gamma=4.21 \times 10^{-9} \mathrm{~m}$ (corresponding to water at $9.7 \mathrm{keV}$ ) to retrieve the phase map.

\subsection{Modified local retrieval}

Both the reverse projection and single image retrieval methods aim primarily at obtaining a reduction in CT scan times, an invaluable attribute especially for clinical or pre-clinical research. However, with current commercial Xray source technologies, phase CT scans on a time-scale of minutes will inevitably come at the cost of sacrificing some degree of spatial resolution. One of the advantages of EI is its ability to obtain resolution which is significantly higher than the detector pixel's size, as the intrinsic resolution is dictated to first approximation by the sample mask's aperture size. ${ }^{20}$ To fully exploit this enhanced resolution, the sample is dithered, and the imaging sequence is repeated for each dithering step. This has the effect of increasing scan times by a factor equal to the number of dithering steps, thus making the imaging setup more sensitive to any time-varying system components (for example environmental changes such as temperature fluctuations and vibrations). By acquiring illumination curves over three days and analysing their features, we have found that the position of the mean of the IC shifted by a considerable amount over time, demonstrating that these effects are indeed significant in our laboratory. ${ }^{32}$ Furthermore, the effect of masks misalignment/defects on reconstructed phase slices was investigated via a wave-optics simulation, ${ }^{33}$ and was shown to lead to ring and gradient artefacts. ${ }^{32}$ In order to address these issues with the aim of providing high-resolution, quantitatively accurate CT reconstructions, a previous phase retrieval method ${ }^{34}$ was modified and adapted to CT scans (modified local retrieval), specifically with additional terms to correct for time-induced changes in the system geometry. ${ }^{32}$ For experimental validation, a biological sample (rat's heart, freeze-dried) was scanned in our laboratory. The experimental setup was similar to the one described above for the reverse projection scan, i.e. the same source, detector and relative distances were used. However, while the masks' periods were the same as before (79 and $98 \mu \mathrm{m}$ for the sample and detector masks, respectively), their aperture sizes were $10 \mu m$ (sample mask) and $17 \mu m$ (detector mask). The source was operated at $25 \mathrm{~mA}$ and $40 \mathrm{kVp}$. The scan consisted of 360 views over $180^{\circ}$, with 6 dithering steps per view corresponding to a resolution of approximately $13 \mu \mathrm{m}$ in the $\mathrm{x}$-direction, ${ }^{20}$ and 3 images per dithering step (for the retrieval of separate absorption, refraction and scattering projections). Flat field images (without a sample) were acquired at each rotation angle, however these are not required for the modified local retrieval. The performance of the modified local retrieval method was tested by comparing CT reconstructions of the experimental dataset using both the newly proposed approach, and a previously used method which assumes perfect alignment ("global retrieval"). ${ }^{15}$ 


\section{RESULTS}

The images shown in Fig. 3 show that the reverse projection method is indeed applicable to EI CT. Moreover, for scans with sample rotation over $180^{\circ}$, the previously used phase retrieval method often results in a gradient seen in the tomogram (Fig. 3(a)). This gradient in grey values is the result of an incorrectly-retrieved offset in the differential phase signal, which is integrated for the purpose of reconstructing a CT slice of $\delta$, the real part of the complex refractive index, therefore leading to inaccuracies in the retrieved $\delta$ values. By employing the reverse projection method instead, the gradient in the tomogram disappears thanks to the averaging effect resulting from the use of projections separated by $180^{\circ}$ (see Fig. 3(b)). By eliminating the time spent on sample mask movement between projections, in combination with the use of a fast read-out detector, virtually all dead times can be eliminated and scan times coincide with the total exposure time, which in this case was 14.4 minutes.
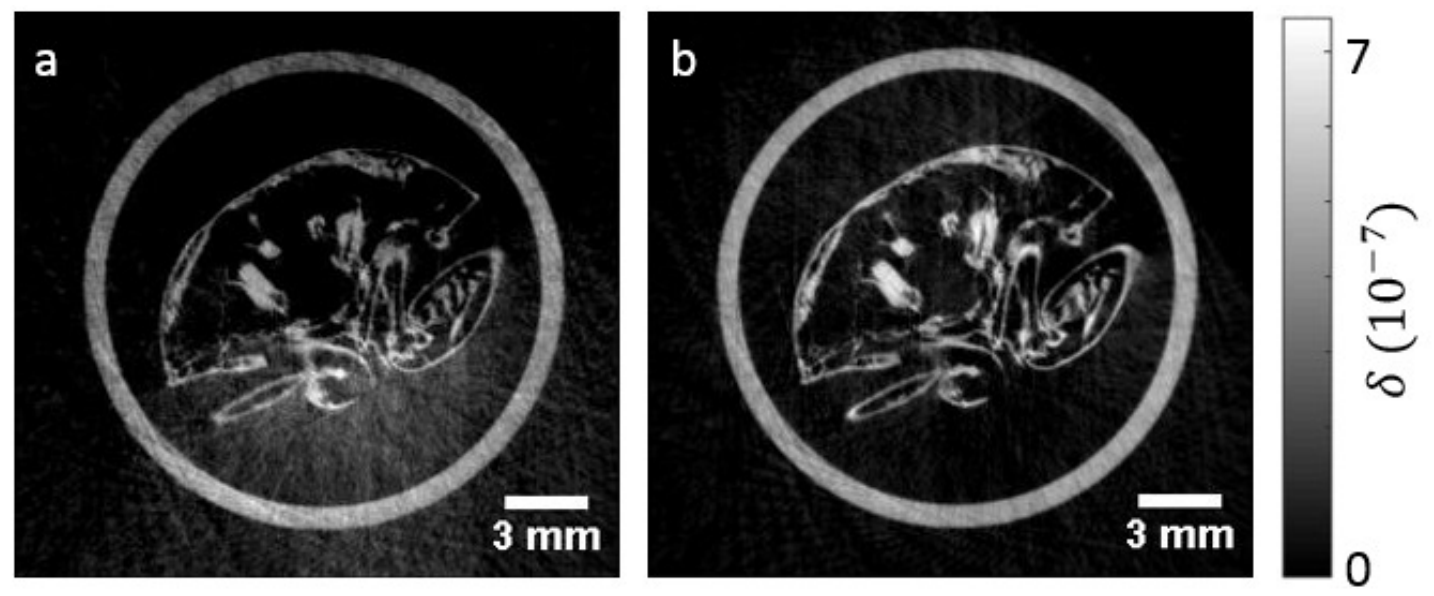

Figure 3. Phase tomograms of a dung beetle, acquired with the laboratory implementation of EI. A qualitative comparison between reconstruction using the conventional two-image phase retrieval method (a), and using the reverse projection approach (b). Apart from leading to shorter scan times, the reverse projection method has the additional advantage of removing the gradient seen across the slice in (a) which is typical for scans acquired over $180^{\circ}$.

Although tested only on planar images so far, the single image phase retrieval algorithm shows promising results. As can be seen in Fig. 4, the retrieved image of the bamboo sample clearly depicts its different layers and structures. In fact, this retrieval algorithm transforms the EI differential contrast (strongly highlighting boundaries between objects) into an area contrast, which is often easier to interpret and can be used directly in a CT reconstruction algorithm. Here, we achieved high image quality by assuming a $\gamma$ value corresponding to that of water, since the exact value for bamboo was not known. Although this results in the method not being fully quantitative, for samples made of unknown materials, $\gamma$ can be tuned until image quality is maximized. The method is also robust against noise, since the algorithm acts as a low-pass filter, ${ }^{27}$ and indeed the commonly observed stripe artefacts (related to the integration of the differential phase signal) are not seen here. 


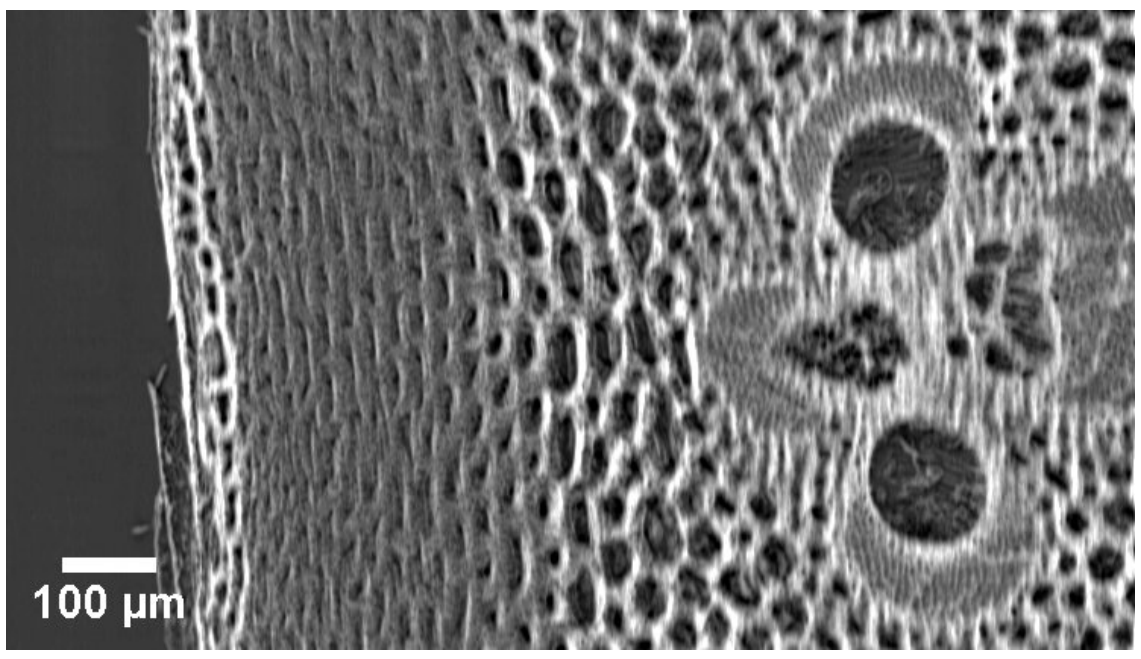

Figure 4. Retrieved phase image of a bamboo sample, from an EI image using the single-image approach. Sample structures are clearly visible due to the resulting area contrast.
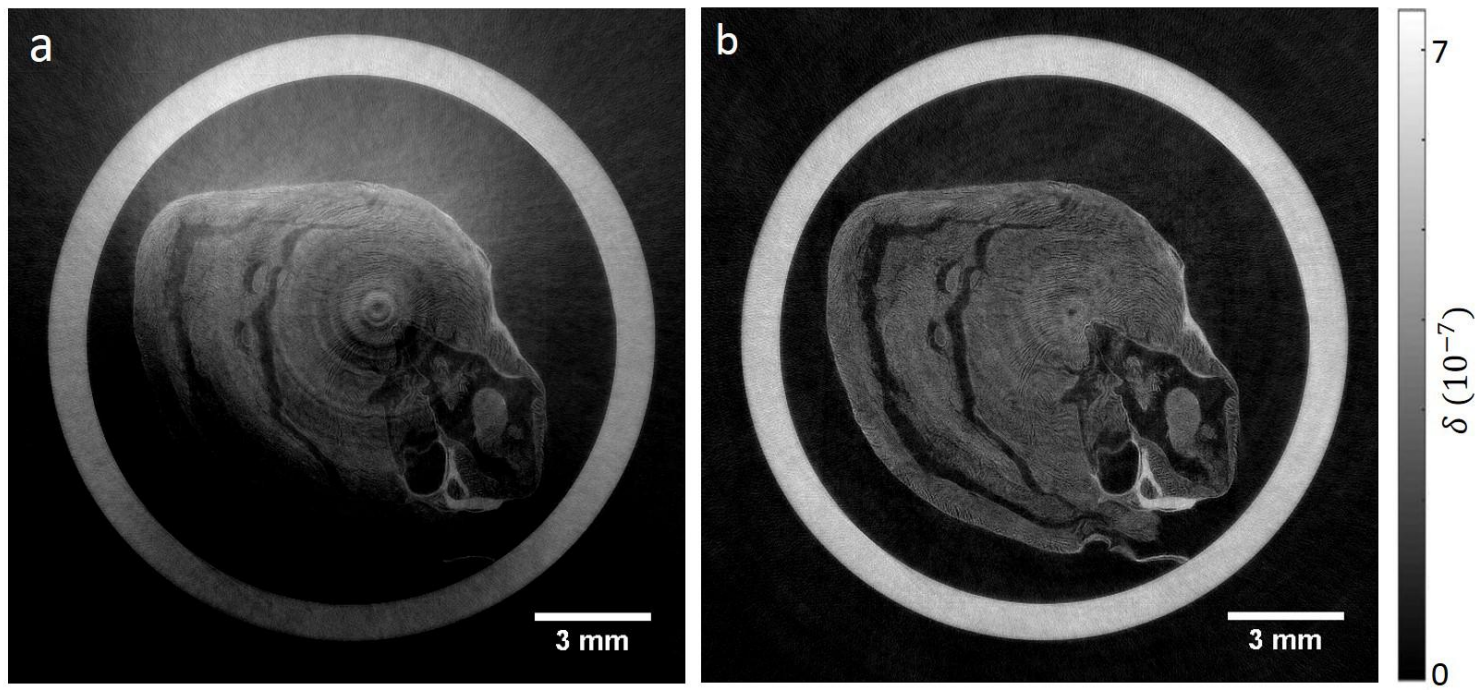

Figure 5. Transverse phase slices of a freeze-dried rat's heart, acquired using the laboratory implementation of EI. The image on the left (a) is the result of using the global retrieval, assuming ideal system alignment, while the image on the right (b) was reconstructed using the modified local retrieval, which corrects for misalignment and time-varying system components.

For the case of high-resolution, long scans, the modified local retrieval approach provided a significant improvement in image quality. As demonstrated in Fig. 5, the use of this algorithm led to the removal of major artefacts (rings, gradient). These artefacts (seen in panel a) are caused by the assumptions made when using the global retrieval, which does not take into account masks' imperfections and misalignment, either present from the beginning or appearing during the scan. These variations are indeed substantial and, when not considered, can lead to a complete loss of sample information (see bottom-left part of the heart in panel a), which are fully recovered when the proposed algorithm is used. Furthermore, while a flat-field image is acquired at each rotation angle for use in the global retrieval, the modified local retrieval does not require any flat field images 
for processing, leading to a significantly reduced scan time.

\section{CONCLUSIONS}

All three methods described here are part of our current work to optimize EI CT, such that it can be performed in different imaging environments, and for a variety of different applications. We have discussed a new acquisition method, "reverse projection", which enables a reduction of scan time by removing the need to reposition the sample mask between acquired frames, therefore allowing a continuous sample rotation. We presented experimental results of a CT scan of a beetle, using the EI laboratory setup, with a total exposure time of approximately 14 minutes.

Scan times could be further reduced by using the "single-image" retrieval algorithm. This algorithm is able to retrieve the phase map of a sample using only one input frame (as opposed to the two frames previously required), provided that the sample is homogeneous and of known material (for a sample of unknown material, an image proportional to the phase map can be retrieved if the ratio between the real and imaginary parts of its complex refractive index is constant). As the algorithm is developed on the basis of this assumption, it is expected to perform well for samples which satisfy this requirement, while it cannot be used for others (e.g. samples containing bone and soft tissue). In addition, the algorithm has been shown to be robust against noise, while also providing images without the common "stripe" artefacts associated with integrating a differential signal. A more in depth analysis of the algorithm's noise transfer properties was recently investigated by Diemoz et al. ${ }^{35}$ Here, we have shown a planar image of a bamboo sample, scanned using synchrotron radiation, and retrieved with the proposed algorithm. Work is currently underway to adapt the proposed algorithm to the laboratory EI configuration, and we expect that a CT implementation of the method could lead to considerably faster CT scans.

Finally, for the case of high-resolution EI CT scans in the laboratory, we have presented the modified local retrieval. This algorithm was developed with the aim of reducing image artefacts associated with system instabilities encountered during long scans. We have presented and compared phase tomograms of a biological sample (rat's heart) acquired in the laboratory, retrieved with both a previously used algorithm, and the modified local retrieval. These demonstrated the complete removal of major image artefacts by use of the algorithm, therefore increasing quantitative accuracy and image quality. It should be noted, for reference, that phase CT results of a rat's heart, acquired using synchrotron radiation, were previously published in studies with the aim of comparing the GI and propagation-based phase techniques. ${ }^{36,37}$ However, in the work presented here, the heart itself was not the focus of the study, and instead it was used as an example for a complex biological object imaged in a laboratory environment, to assess the performance of the proposed phase retrieval algorithm. In addition, while demonstrated here on a small sample, this algorithm is expected to perform equally well on larger samples, as the computation is performed on a pixel-by-pixel basis. Therefore, when considering samples which require high-resolution $(\sim 10 \mu \mathrm{m})$ and for which dose considerations are less stringent (e.g. material science, ex-vivo specimen imaging), this approach can provide accurate and artefact-free CT scans in non-ideal environments.

With regards to the potential for dose reduction, out of the three approaches presented in this paper, the "single-image" method is the one which is likely to lead to the highest reduction in dose. However, since the method has not yet been applied to CT, a comparison between the obtained signal-to-noise ratio using the proposed method and the two-image retrieval, has not been done. Therefore, the use of a single image does not necessarily imply a reduction of dose by a factor of two, and so further investigation is required. It is worth noting that while we anticipate the single-image retrieval to lead to a reduction in dose, we have previously shown that it is possible to perform low-dose EI CT in the laboratory for thin objects using the two-image retrieval. ${ }^{19}$

\section{ACKNOWLEDGMENTS}

This work was supported by the UK Engineering and Physical Sciences Research Council (EP/I021884/1). ME was supported by the Royal Academy of Engineering under the RAEng Research Fellowships scheme. PCD is supported by a Marie Curie Career Integration Grants PCIG12-GA-2012-333990 within the Seventh Framework Programme of the European Union. PDC and LU were funded by the OAK Foundation (OCAY-14-191) and the 
GOSHCC (V0615, V1282), and supported by the NIHR Biomedical Research Centre at GOSH NHS Foundation Trust and UCL (RP-2014-04-046).

\section{REFERENCES}

[1] Bravin, A., Coan, P., and Suortti, P., "X-ray phase-contrast imaging: from pre-clinical applications towards clinics.," Phys. Med. Biol. 58(1), R1-35 (2013).

[2] Wilkins, S. W., Nesterets, Y. I., Gureyev, T. E., Mayo, S. C., Pogany, A., and Stevenson, A. W., "On the evolution and relative merits of hard X-ray phase-contrast imaging methods," Phil. Trans. R. Soc. 372, 20130021 (2014).

[3] Olivo, A., Arfelli, F., Cantatore, G., Longo, R., Menk, R. H., Pani, S., Prest, M., Poropat, P., Rigon, L., Tromba, G., Vallazza, E., and Castelli, E., "An innovative digital imaging set-up allowing a low-dose approach to phase contrast applications in the medical field," Med. Phys. 28(8), 1610 (2001).

[4] Olivo, A. and Speller, R., "A coded-aperture technique allowing x-ray phase contrast imaging with conventional sources," Appl. Phys. Lett. 91(7), 074106 (2007).

[5] Olivo, A., Diemoz, P. C., and Bravin, A., "Amplification of the phase contrast signal at very high x-ray energies," Opt. Lett. 37(5), 915-917 (2012).

[6] Diemoz, P. C., Endrizzi, M., Zapata, C. E., Pešić, Z. D., Rau, C., Bravin, A., Robinson, I. K., and Olivo, A., "X-ray phase-contrast imaging with nanoradian angular resolution," Phys. Rev. Lett. 110(13), 138105 (2013).

[7] Munro, P. R. T., Ignatyev, K., Speller, R. D., and Olivo, A., "Phase and absorption retrieval using incoherent X-ray sources," Proc. Natl. Acad. Sci. 109(35), 13922-13927 (2012).

[8] Diemoz, P. C., Hagen, C. K., Endrizzi, M., and Olivo, A., "Sensitivity of laboratory based implementations of edge illumination X-ray phase-contrast imaging," Appl. Phys. Lett. 103(24), 244104 (2013).

[9] Endrizzi, M., Diemoz, P. C., Millard, T. P., Louise Jones, J., Speller, R. D., Robinson, I. K., and Olivo, A., "Hard X-ray dark-field imaging with incoherent sample illumination," Appl. Phys. Lett. 104(2), 024106 (2014).

[10] Pfeiffer, F., Weitkamp, T., Bunk, O., and David, C., "Phase retrieval and differential phase-contrast imaging with low-brilliance X-ray sources," Nat. Phys. 2(4), 258-261 (2006).

[11] Momose, A., Yashiro, W., Kido, K., Kiyohara, J., Makifuchi, C., Ito, T., Nagatsuka, S., Honda, C., Noda, D., Hattori, T., Endo, T., and Tanaka, J., "X-ray phase imaging: from synchrotron to hospital," Phil. Trans.R.Soc. A. 372, 20130023 (2014).

[12] Olivo, A., Bohndiek, S. E., Griffiths, J. A., Konstantinidis, A., and Speller, R. D., "A non-free-space propagation x-ray phase contrast imaging method sensitive to phase effects in two directions simultaneously," Appl. Phys. Lett. 94(4), 044108 (2009).

[13] Kallon, G. K., Wesolowski, M., Vittoria, F. A., Endrizzi, M., Basta, D., Millard, T. P., Diemoz, P. C., and Olivo, A., "A laboratory based edge-illumination x-ray phase-contrast imaging setup with two-directional sensitivity," Appl. Phys. Lett. 107(20), 103-108 (2015).

[14] Munro, P., Hagen, C., Szafraniec, M., and Olivo, A., "A simplified approach to quantitative coded aperture X-ray phase imaging.," Opt. Express 21(9), 11187-201 (2013).

[15] Endrizzi, M. and Olivo, A., "Absorption, refraction and scattering retrieval with an edge-illumination-based imaging setup," J. Phys. D. Appl. Phys. 47(50), 505102 (2014).

[16] Hagen, C. K., Diemoz, P. C., Endrizzi, M., Rigon, L., Dreossi, D., Arfelli, F., Lopez, F. C. M., Longo, R., and Olivo, A., "Theory and preliminary experimental verification of quantitative edge illumination x-ray phase contrast tomography.," Opt. Express 22(7), 7989-8000 (2014).

[17] Hagen, C. K., Diemoz, P. C., Endrizzi, M., and Olivo, A., "The effect of the spatial sampling rate on quantitative phase information extracted from planar and tomographic edge illumination x-ray phase contrast images," J. Phys. D. Appl. Phys. 47(45), 455401 (2014).

[18] Munro, P. and Olivo, A., "X-ray phase-contrast imaging with polychromatic sources and the concept of effective energy," Phys. Rev. A 87(5), 053838 (2013).

[19] Hagen, C. K., Munro, P. R. T., Endrizzi, M., Diemoz, P. C., and Olivo, A., "Low-dose phase contrast tomography with conventional x-ray sources.," Med. Phys. 41(7), 070701 (2014). 
[20] Diemoz, P., Vittoria, F., and Olivo, A., "Spatial resolution of edge illumination X-ray phase-contrast imaging.," Opt. Express 22(13), 15514-29 (2014).

[21] Olivo, A., Ignatyev, K., Munro, P., and Speller, R., "Design and realization of a coded-aperture based X-ray phase contrast imaging for homeland security applications," Nucl. Instruments Methods Phys. Res. Sect. A 610(2), 604-614 (2009).

[22] Olivo, A., Gkoumas, S., Endrizzi, M., Hagen, C. K., Szafraniec, M. B., Diemoz, P. C., Munro, P. R. T., Ignatyev, K., Johnson, B., Horrocks, J. A., Vinnicombe, S. J., Jones, J. L., and Speller, R. D., "Low-dose phase contrast mammography with conventional x-ray sources.," Med. Phys. 40(9), 090701 (2013).

[23] Hagen, C. K., Maghsoudlou, P., Totonelli, G., Diemoz, P. C., Endrizzi, M., Rigon, L., Menk, R.-H., Arfelli, F., Dreossi, D., Brun, E., Coan, P., Bravin, A., De Coppi, P., and Olivo, A., "High contrast microstructural visualization of natural acellular matrices by means of phase-based x-ray tomography.," Sci. Rep. 5, 18156 (2015).

[24] Endrizzi, M., Murat, B. I. S., Fromme, P., and Olivo, A., "Edge-illumination X-ray dark-field imaging for visualising defects in composite structures," Compos. Struct. 134, 895-899 (2015).

[25] Wang, M., Zhu, P.-P., Zhang, K., Hu, X.-F., Huang, W.-X., Cen, Y.-W., Yuan, Q.-X., Yu, X.-L., and Wang, J.-Y., "A new method to extract angle of refraction in diffraction enhanced imaging computed tomography," J. Phys. D. Appl. Phys. 40(22), 6917-6921 (2007).

[26] Hagen, C. K., Endrizzi, M., Diemoz, P. C., and Olivo, A., "Reverse projection retrieval in edge illumination x-ray phase contrast computed tomography," J. Phys. D. Appl. Phys. 49, 255501 (2016).

[27] Diemoz, P. C., Vittoria, F. a., Hagen, C. K., Endrizzi, M., Coan, P., Brun, E., Wagner, U. H., Rau, C., Robinson, I. K., Bravin, A., and Olivo, A., "Single-image phase retrieval using an edge illumination X-ray phase-contrast imaging setup," J. Synchrotron Radiat. 22(4), 1072-1077 (2015).

[28] Paganin, D., Mayo, S. C., Gureyev, T. E., Miller, P. R., and Wilkins, S. W., "Simultaneous phase and amplitude extraction from a single defocused image of a homogeneous object.," J. Microsc. 206(Pt 1), 33-40 (2002).

[29] Pavlov, K. M., Gureyev, T. E., Paganin, D., Nesterets, Y. I., Morgan, M. J., and Lewis, R. A., "Linear systems with slowly varying transfer functions and their application to x-ray phase-contrast imaging," $J$. Phys. D. Appl. Phys. 37(19), 2746-2750 (2004).

[30] Briedis, D., Siu, K. K. W., Paganin, D. M., Pavlov, K. M., and Lewis, R. A., "Analyser-based mammography using single-image reconstruction.," Phys. Med. Biol. 50(15), 3599-3611 (2005).

[31] Vittoria, F. A., Endrizzi, M., Diemoz, P. C., Wagner, U. H., Rau, C., Robinson, I. K., and Olivo, A., "Virtual edge illumination and one dimensional beam tracking for absorption, refraction, and scattering retrieval," Appl. Phys. Lett. 104(13), 134102 (2014).

[32] Zamir, A., Endrizzi, M., Hagen, C. K., Vittoria, F. A., Urbani, L., De Coppi, P., and Olivo, A., "Robust phase retrieval for high resolution edge illumination x-ray phase-contrast computed tomography in non-ideal environments," Sci. Rep. 6, 31197 (2016).

[33] Vittoria, F. a., Diemoz, P. C., Endrizzi, M., Rigon, L., Lopez, F. C., Dreossi, D., Munro, P. R. T., and Olivo, A., "Strategies for efficient and fast wave optics simulation of coded-aperture and other x-ray phase-contrast imaging methods," Appl. Opt. 52(28), 6940-6947 (2013).

[34] Endrizzi, M., Basta, D., Olivo, A., Endrizzi, M., Basta, D., and Olivo, A., "Laboratory-based X-ray phasecontrast imaging with misaligned optical elements," Appl. Phys. Lett. 107(12), 124103 (2015).

[35] Diemoz, P. C., Vittoria, F. A., Hagen, C. K., Endrizzi, M., Coan, P., Bravin, A., Wagner, U. H., Rau, C., Robinson, I. K., and Olivo, A., "A single-image retrieval method for edge illumination x-ray phase-contrast imaging: application and noise analysis," Phys. Medica (2016, in press).

[36] Zanette, I., Lang, S., Rack, A., Dominietto, M., Langer, M., Pfeiffer, F., Weitkamp, T., and Müller, B., "Holotomography versus X-ray grating interferometry : A comparative study," Appl. Phys. Lett. 103, 244105 (2013).

[37] Lang, S., Zanette, I., Dominietto, M., Langer, M., Rack, A., Schulz, G., Duc, G. L., David, C., Mohr, J., Pfeiffer, F., and Weitkamp, T., "Experimental comparison of grating- and propagation-based hard X-ray phase tomography of soft tissue," J. Appl. Phys. 116, 154903 (2014). 